\title{
Oral mucositis associated with anti-EGFR therapy in colorectal cancer: single institutional retrospective cohort study
}

\author{
Satoshi Dote ${ }^{1,2^{*}} \mathbb{D}$, Shoji Itakura ${ }^{3}$, Kohei Kamei ${ }^{1}$, Daiki Hira ${ }^{2,4}$, Satoshi Noda ${ }^{2}$, Yuka Kobayashi' and Tomohiro Terada ${ }^{2}$
}

\begin{abstract}
Background: Chemotherapy-induced oral mucositis impairs the quality of life. The difference in severity of oral mucositis between different anti-epidermal growth factor receptor (EGFR) antibodies combined with cytotoxic drugs in colorectal cancer is unclear. The aim of this study was to investigate the differences in oral mucositis between panitumumab (Pmab) and cetuximab (Cmab) combined with 5-fluorouracil (5-FU).
\end{abstract}

Methods: We conducted a retrospective cohort study. A total of 75 colorectal cancer outpatients treated with an antiEGFR antibody combined with FOLFOX, FOLFIRI, or 5-FU/leucovorin as the first- to third-line treatment were included. The primary endpoint was the incidence of grade 2-3 oral mucositis. The secondary endpoint was the time to onset of oral mucositis. We also compared the incidence of toxicities of interest, skin toxicity, hypomagnesaemia and neutropenia, and time to treatment failure (TTF) between the two groups.

Results: Thirty-two patients treated with Pmab and 43 patients treated with Cmab were evaluated. Patient characteristics were similar between the two groups. The incidence of grade 2-3 oral mucositis was significantly higher with Pmab than with Cmab (31.3\% vs 9.3\%, $P<0.05)$. Moreover, the incidence of grade 3 oral mucositis was significantly higher in patients treated with Pmab (18.8\% vs 0\%, $P<0.01)$. The mean (SD) cycles to onset of the worst oral mucositis was $3.0(2.9)$ in the Pmab group and $2.3(1.7)$ in the Cmab group $(P=0.29)$. Oral mucositis was characterized by glossitis and cheilitis. The incidences of other toxicities were the following (Pmab vs $C$ mab): grade $2-3$ skin toxicity: $68.8 \%$ vs $74.4 \%(P=0.61)$, grade 2-3 hypomagnesaemia: $9.3 \%$ vs $7.0 \%(P=1.00)$, grade $3-4$ neutropenia: $28.1 \%$ vs $37.2 \%(P=0.46)$. The median TTF was not significantly different, i.e., 223 days vs 200 days $(P=0.39)$ for Pmab vs $C$ mab.

Conclusions: Pmab-based chemotherapy resulted in significantly higher grades of oral mucositis compared with Cmabbased chemotherapy. The oral condition should be monitored carefully and early supportive care should be provided for patients treated with Pmab-based chemotherapy.

Keywords: Oral mucositis, Colorectal cancer, Panitumumab, Cetuximab, Anti-EGFR antibody, 5-fluorouracil

\section{Background}

Oral mucositis refers to mucosal damage secondary to cancer therapy occurring in the oral cavity, and can be caused by both chemotherapy and radiotherapy [1-3]. Oral mucositis presents as erythema and/ or ulceration of the oral mucosa. It is typically painful, requiring analgesics, leading to alteration in

\footnotetext{
* Correspondence: dote@belle.shiga-med.ac.jp

'Department of Pharmacy, Kyoto-Katsura Hospital, 17, Yamadahiraocho, Kyoto-shi Nishikyo-ku, Kyoto 615-8256, Japan

2Department of Pharmacy, Shiga University of Medical Science Hospital, Seta

Tsukinowa-cho, Otsu, Shiga 520-2192, Japan

Full list of author information is available at the end of the article
}

cancer therapy, risk for infection, and it impairs nutritional intake and quality of life [1-4].

Epidermal growth factor (EGF) regulates epithelial cell proliferation, growth, and migration, is present in biological fluids, including saliva, and plays an important role in maintaining the epithelial barrier and healing damaged mucosa [5]. Regarding the role of EGF in oral mucosa in oncology, salivary EGF levels have been reported to be associated with the severity of oral mucositis induced by radiation therapy $[6,7]$. Furthermore, Kim et al. reported that recombinant human EGF oral spray improved 
mucotoxic regimen-induced oral mucositis in patients undergoing hematopoietic stem cell transplant [8]. Anti-epidermal growth factor receptor (EGFR) antibodies, panitumumab (Pmab) and cetuximab (Cmab), are widely used for patients with wild-type (WT) KRAS metastatic colorectal cancer [9-11]. The toxicities of anti-EGFR antibodies were characterized by skin toxicity $[12,13]$, infusion reaction [14-16], electrolyte imbalance [16-18], and interstitial pneumonia $[16,19]$. It was also reported that the incidence of oral mucositis was 5 to $7 \%$ when an anti-EGFR antibody was used as monotherapy [20]. Interestingly, the incidence of oral mucositis was higher (all grades: about $30-40 \%$, grade 3 or higher: approximately $10 \%$ ) when the anti-EGFR antibody was used in combination with 5-fluorouracil (5-FU) [21-25], which is a well-known mucotoxic drug [26]. Although the mechanism of oral mucositis induced by the anti-EGFR antibody concomitant with 5-FU was not clarified, anti-EGFR therapy may deteriorate 5 -FU-induced oral mucositis by interfering with the wound healing process due to blockage of EGF.

In Japan, board-certified oncology pharmacists provide pharmaceutical care for oncology outpatients [27, 28]. In our institute, board-certified oncology pharmacists routinely check the oral condition in outpatients as a part of pharmaceutical care. To date, in one comparative phase III study, there was not a significant difference in the incidence of oral mucositis between Pmab and Cmab used as monotherapy [20]. However, head-to-head studies comparing the incidence of oral mucositis between Pmab- and Cmab-combined with 5-FU have not been reported. Therefore, we conducted a retrospective cohort study to examine the incidence and severity of oral mucositis in patients who were receiving anti-EGFR antibodies concomitantly with 5-FU.

\section{Methods}

\section{Study design}

Study design was retrospective, single institutional cohort study. Eligible patients were metastatic colorectal cancer outpatients treated with an anti-EGFR antibody combined with FOLFOX: infusional 5-FU plus leucovorin (5-FU/LV) with the addition of oxaliplatin, FOLFIRI: 5-FU/LV with the addition of irinotecan, or 5 -FU/LV as the first- to third-line treatment at Kyoto-Katsura Hospital (details of chemotherapy are shown in Additional file 1: Table S1). If the patients were treated with both Pmab and Cmab until the third-line of chemotherapy, we allocated the patient to the prior anti-EGFR antibody group. Exclusion criteria were 5-FU-free chemotherapy treatment and inpatients. The periods of recruitment and data collection (follow-up) from electronic medical records were January 1, 2012 to February 28, 2017 and January 1, 2012 to March 31, 2017, respectively. This study was performed in accordance with the Declaration of Helsinki and its amendments, and the protocol was approved by the Ethics Committee of Kyoto-Katsura Hospital (Approval number: 501).

\section{Procedures}

We divided the eligible patients into two groups. The Pmab group included the patients treated with Pmab-based chemotherapy and the Cmab group included the patients treated with Cmab-based chemotherapy. The primary endpoint was the incidence of grade 2-3 oral mucositis (Table 1) in either groups. At our institute, board-certified oncology pharmacists and nurses catch adverse events by carefully interviewing while referring to the medical questionnaire answered by the patient and correctly recording the grade of toxicities in the electronic medical records. Oral mucositis was graded at each outpatient chemotherapy session by physicians, board-certified oncology pharmacists, and nurses. The secondary endpoint was the time to onset of oral mucositis, defined as the cycle when oral mucositis occurred after initiation of anti-EGFR therapy. A cycle was defined in biweekly intervals. If $\mathrm{Cmab}$ was given weekly, one cycle contained two Cmab infusions. The number of cycles at the first onset of any grade of oral mucositis and that at the onset of the worst grade of oral mucositis for each patient were compared between the two groups. Once anti-EGFR therapy was initiated, we counted the number of cycles regardless of whether anti-EGFR antibody administration was postponed or discontinued due to toxicity during chemotherapy. We also compared toxicities of interest between the two groups: the incidence of skin toxicity, neutropenia, and hypomagnesaemia, and time to treatment failure (TTF), which was defined as the time from treatment initiation to discontinuation for any reason, including disease progression, treatment toxicities, patient preference, or death. Any toxicities

Table 1 National Cancer Institute Common Terminology Criteria for Adverse Events v4.0 "Oral mucositis"

\begin{tabular}{llll}
\hline Grade 1 & Grade 2 & Grade 3 & Grade 4 \\
\hline $\begin{array}{l}\text { Asymptomatic or mild } \\
\text { symptoms; intervention not }\end{array}$ & Moderate pain; not & Severe pain; & Grade 5 \\
indicated & $\begin{array}{l}\text { interfering with oral } \\
\text { intake; modified diet } \\
\text { indicated }\end{array}$ & intake & $\begin{array}{l}\text { consequence; urgent } \\
\text { intervention } \\
\text { indicated }\end{array}$ \\
\hline
\end{tabular}


were graded according to the Common Terminology Criteria for Adverse Events (CTCAE) version 4.0.

\section{Statistical analyses}

Binary outcomes were compared with the Fisher's exact test, and continuous outcomes with the unpaired Student's t-test, and time-to-event data was compared by the log-rank test using the Kaplan-Meier method. JMP 9 software (SAS Institute, Japan) was used for all analyses and a $p$-value less than 0.05 was regarded as significant. The heterogeneity of the treatment effects on the primary endpoint was assessed for five pre-specified subgroups. Subgroups were based on history of diabetes and smoking status as risk factors for oral mucositis [3], and sex, treatment regimen, and line of treatment, which likely affect the incidence of oral mucositis. When patients underwent curative operations, such as hepatectomy or primary tumor resection, and radiotherapy during anti-EGFR antibody combined with 5-FU chemotherapy, the patient was treated as a censored case in the time-to-event analysis.

\section{Results \\ Participants}

Thirty-two patients were evaluated in the Pmab group and 43 patients were evaluated in the Cmab group. Patient characteristics are shown in Table 2 . There were no significant differences in patient demographics between the two groups. Most patients were treated with the anti-EGFR antibody combined with 5-FU as first- or second-line chemotherapy and had a good performance status.

\section{The incidence of oral mucositis}

The incidence of oral mucositis between the two groups is shown in Fig. 1. The primary outcome, the incidence of grade $2-3$ oral mucositis, was significantly higher in

Table 2 Patient characteristics

\begin{tabular}{|c|c|c|c|}
\hline & Pmab group $N=32$ & Cmab group $N=43$ & $P$-value \\
\hline \multicolumn{4}{|l|}{ Sex } \\
\hline Male & $23(72 \%)$ & $30(70 \%)$ & 1.00 \\
\hline Age (years) & $65.7(9.0)$ & $63.1(12.4)$ & 0.33 \\
\hline Body weight (kg) & $58.1(10.0)$ & $57.9(9.0)$ & 0.91 \\
\hline Body surface area $\left(\mathrm{kg} / \mathrm{m}^{2}\right)$ & $1.62(0.17)$ & $1.60(0.16)$ & 0.59 \\
\hline \multicolumn{4}{|l|}{ Performance Status (ECOG) } \\
\hline $0 / 1 / 2$ & $15(47 \%) / 16(50 \%) / 1$ (3\%) & 27 (63\%) / 15 (35\%) / 1 (2\%) & 0.39 \\
\hline Diabetes mellitus & $8(25 \%)$ & $6(14 \%)$ & 0.25 \\
\hline \multicolumn{4}{|l|}{ Smoking status } \\
\hline Never / former / current & $8(25 \%) / 15$ (47\%) / 9 (28\%) & 17 (40\%) / 16 (37\%) / 10 (23\%) & 0.42 \\
\hline Serum albumin level (g/dl) & $3.64(0.66)$ & $3.74(0.36)$ & 0.43 \\
\hline \multicolumn{4}{|l|}{ Primary origin of tumors } \\
\hline Rectal / colon / other & $9(28 \%) / 22(69 \%) / 1(3 \%)$ & 22 (51\%) / $18(42 \%) / 3$ (7\%) & 0.07 \\
\hline $\begin{array}{l}\text { On the left side of the colon / on } \\
\text { the right side of the colon }\end{array}$ & $21(66 \%) / 11(34 \%)$ & $33(78 \%) / 10(23 \%)$ & 0.31 \\
\hline \multicolumn{4}{|l|}{ Line of treatment } \\
\hline 1st / 2nd / 3rd & $25(78 \%) / 6$ (19\%) / 1 (3\%) & $38(88 \%)$ / 4 (9\%) / 1 (2\%) & 0.47 \\
\hline \multicolumn{4}{|l|}{ Combined regimen } \\
\hline FOLFOX / FOLFIRI / LV5FU & $22(69 \%) / 9(28 \%) / 1$ (3\%) & $38(88 \%) / 4$ (9\%) / 1 (2\%) & 0.10 \\
\hline \multicolumn{4}{|l|}{ Concomitant bolus 5-FU } \\
\hline Presence / absence & $32(100 \%) / 0(0 \%)$ & $41(95 \%) / 2(5 \%)$ & 0.50 \\
\hline Relative dose intensity in cycle 1 (\%) & $97.3(7.8)$ & $93.0(21.6)$ & 0.28 \\
\hline \multicolumn{4}{|l|}{ Cetuximab interval $^{\mathrm{b}}$} \\
\hline Weekly / biweekly & - & $13(30 \%) / 30$ (70\%) & - \\
\hline
\end{tabular}

Data are expressed as mean (SD) and $\mathrm{n}(\%)$.

${ }^{a}$ On the left side of the colon means descending colon, sigmoid colon, and rectum. On the right side of the colon means the cecum and ascending colon. ${ }^{b}$ Weekly means cetuximab administered weekly; the initial dose was $400 \mathrm{mg} / \mathrm{m}^{2}$ and the maintenance dose was $250 \mathrm{mg} / \mathrm{m}^{2}$. Biweekly means a $500-\mathrm{mg} / \mathrm{m}^{2}$ dose of cetuximab administered every other week. 


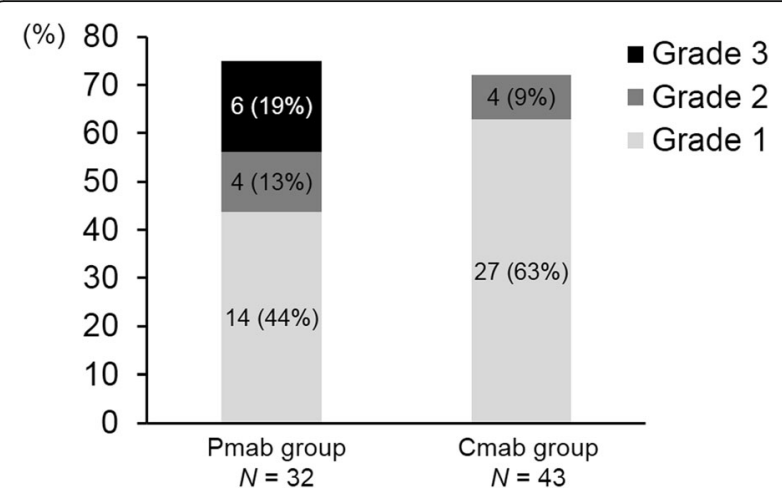

Fig. 1 Primary endpoint: the incidence of grade 2-3 oral mucositis between the Pmab and Cmab groups. The number of patients $(\mathrm{N})$ and the incidence of oral mucositis (\%) of each grade are shown in the bar charts. The incidence of oral mucositis was the following (Pmab group vs Cmab group); All grades: 24 (75\%) vs 31 (72\%), $p>0.05$. grade $2-3: 10(31.3 \%)$ vs $4(9.3 \%), p<0.05$. grade $3: 6(18.8 \%)$ vs $0(0 \%), p<0.01$

the Pmab group than in the Cmab group (31.3\% vs 9.3\%, $p<0.05)$. Moreover, the incidence of grade 3 oral mucositis was significantly higher in the Pmab group than in the Cmab group ( $18.8 \%$ vs $0 \%, p<0.01)$. Grade 2 to 3 oral mucositis in either groups was mainly characterized by glossitis (the tip of the tongue) and cheilitis (the inside of the lower lip). The time to onset of oral mucositis between the two groups is shown in Fig. 2. The mean cycles (SD) to the first onset of any grade of oral mucositis each patient was 1.8 (1.4) in the Pmab group and 2.2 (1.6) in the Cmab group $(p=0.32)$. The mean cycles $(\mathrm{SD})$ to onset of the worst grade of oral mucositis each patient was 3.0 (2.9) in the Pmab group and 2.3 (1.7) in the Cmab group $(p=0.29)$. We also conducted subgroup analysis and calculated the odds ratio for grade 2-3 oral mucositis (Fig. 3). The point estimates of the odds ratio among subgroups were all poor for Pmab-based chemotherapy.

\section{Other toxicities of interest and TTF}

The summary of other toxicities of interest is shown in Table 3. The incidence of toxicities between the two groups did not differ significantly. The median TTF was 223 days in the Pmab group and 200 days in the Cmab group (hazard ratio $0.78,95 \%$ confidence interval 0.42-1.38) (Fig. 4).

\section{Discussion}

We clarified that Pmab-based chemotherapy concomitant with 5-FU could result in a higher incidence of grade 2-3 oral mucositis compared with Cmab-based chemotherapy concomitant with 5-FU. Furthermore, the odds ratio of grade $2-3$ oral mucositis was higher in the Pmab group than in the Cmab group among subgroups. Conversely, other toxicities of interest and TTF were not different between the two groups. When these results of this study were compared with the previous studies, the following differences were found. First, in this study, grade 3 oral mucositis was not observed in the Cmab group. On the other hand, the previous MRC COIN study reported that the incidence of grade 3 or higher was $10 \%$ in patients treated with $\mathrm{Cmab}$ combined with FOLFOX [25]. As the mean half-life of Cmab in the steady state was reported to be $114 \mathrm{~h}$ (about 5 days) [29], the difference in oral toxicity between the MRC COIN study and our study may be due to the treatment interval of $\mathrm{Cmab}$ because the percentage of weekly $\mathrm{Cmab}$ administration was $100 \%$ in the MRC COIN study and 30\% (Table 2) in this study. Second, our study reported a higher incidence (all grades: over 70\%) of oral mucositis than previous studies (all grades: approximately 30\% 40\% [22-24]). We catch adverse events by carefully interviewing referring to the medical questionnaire answered by patient at each outpatient

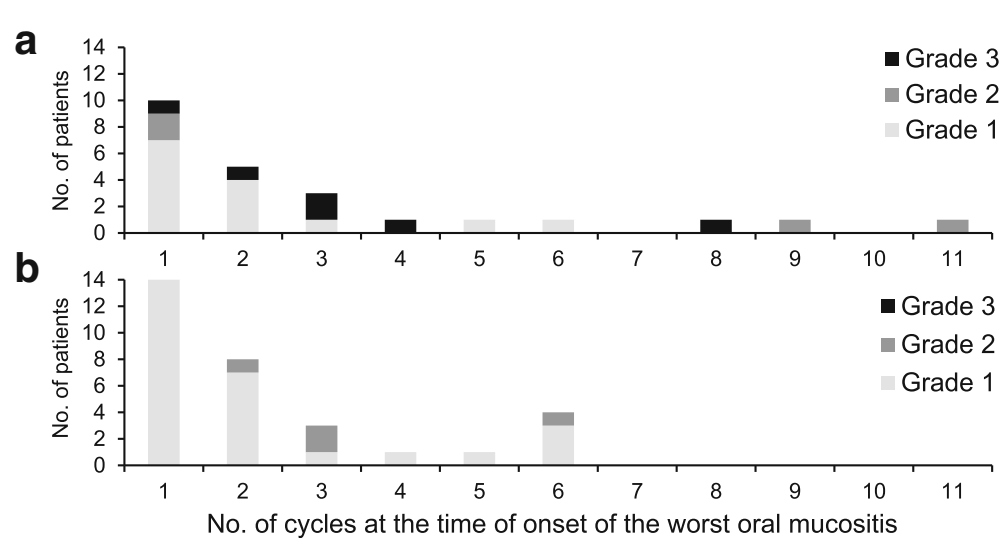

Fig. 2 Secondary endpoint: the time to onset of the worst grade of oral mucositis each patient in the Pmab group (a) and Cmab group (b). The mean (SD) cycles to onset of the worst grade of oral mucositis each patient was 3.0 (2.9) in the Pmab group and 2.3 (1.7) in the Cmab group 


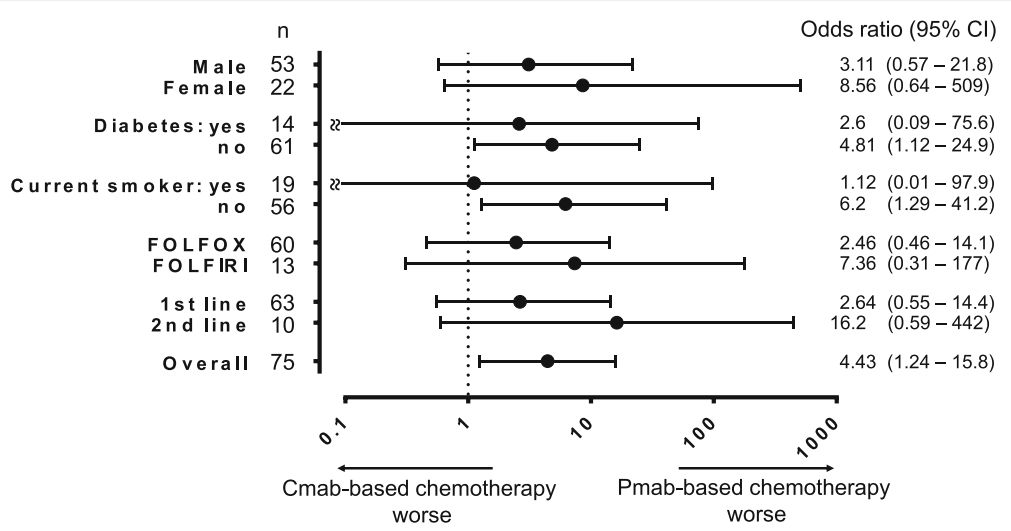

Fig. 3 Odds ratio for grade 2-3 oral mucositis in pre-specified subgroups. Black dots indicate point estimates of the odds ratio and I bars indicate $95 \%$ confidence intervals of the odds ratio. The odds ratio was calculated by adding 0.5 to each value when no grade $2-3$ oral mucositis was observed in each subgroup

chemotherapy session. Therefore, we noted minor oral toxicity and oral pain, which resulted in the high incidence of oral mucositis. Third, although the previous study reported that the incidence of hypomagnesaemia was higher in patients treated with Pmab than in those with Cmab [20], the incidence of hypomagnesaemia did not differ between the two groups in this study. This may be because we administered prophylactic magnesium supplements at each cycle of chemotherapy after the occurrence of grade 1 hypomagnesaemia.

Anti-EGFR antibodies play a role extracellularly and not intracellularly because of their large molecular weight. Therefore, anti-EGFR antibodies mainly distribute in the blood and blood flow-rich tissues such as the kidneys, liver, spleen, and lung [30-32]. A previous study reported that the affinity to EGFR was higher for Pmab (50 pmol/L [33]) than for Cmab (400 pmol/L [34]). Based on this, toxicity in blood flow-rich tissues may likely occur with Pmab. Supporting this hypothesis, in the above mentioned

Table 3 Summary of toxicities of interest

\begin{tabular}{clll}
\hline & $\begin{array}{l}\text { Pmab group } \\
N=32\end{array}$ & $\begin{array}{l}\text { Cmab group } \\
N=43\end{array}$ & $P$-value \\
\hline Skin toxicity & & & \\
All grades & $32(100 \%)$ & $41(95 \%)$ & 0.50 \\
Grade 2-3 & $22(69 \%)$ & $32(74 \%)$ & 0.61 \\
Grade 3 & $12(38 \%)$ & $11(26 \%)$ & 0.32 \\
Hypomagnesaemia & & & \\
All grades & $21(66 \%)$ & $27(63 \%)$ & 1.00 \\
Grade 2-3 & $3(9 \%)$ & $3(7 \%)$ & 1.00 \\
Neutropenia & & & \\
Grade 3-4 & $9(28 \%)$ & $16(37 \%)$ & 0.46 \\
Grade 4 & $2(6 \%)$ & $3(7 \%)$ & 1.00 \\
\hline
\end{tabular}

ASPECCT trial, a randomised phase 3 trial that compared Pmab and Cmab in patients with chemotherapy-refractory WT KRAS exon 2 colorectal cancer, the incidence of grade 3-4 hypomagnesaemia was significantly higher in patients treated with Pmab than in patients treated with Cmab (7\% vs 3\%) [20]. As the kidneys are one of the most blood flow-rich tissues, anti-EGFR antibodies inhibit the renal distal tubule magnesium transporter, a transient receptor potential melastatin type 6 channel that is stimulated by EGF, resulting in hypomagnesaemia [35]. Therefore, due to the rich blood flow in the oral mucosa, the difference in oral toxicity between Pmab and Cmab may be explained by the same hypothesis. In addition, we observed grade 2-3 oral mucositis in both groups at the tip of the tongue and the inside of the lower lip, which are in contact with saliva. As salivary EGF plays an important role in the healing of damaged mucosa induced by radiotherapy $[6,7]$ and chemotherapy [8], mucotoxicity induced by anti-EGFR therapy combined with 5-FU may occur due to blockage of EGF at saliva-rich sites.

The strength of this study is that the incidence of oral mucositis induced by anti-EGFR antibody combined with 5-FU based chemotherapy was reported in the real world setting. At each outpatient chemotherapy session, we routinely assessed the severity of chemotherapy induced-toxicities based on CTCAE version 4.0, as well as toxicities that happened during chemotherapy intervals referring to the medical questionnaire answered by the patients. Therefore, regarding stomatitis, we meticulously interviewed and assessed about the oral condition when patients reported oral mucositis.

Some limitations exist in this study. First, we could not perform multivariate analysis for the primary endpoint considering covariates, such as history of diabetes and 


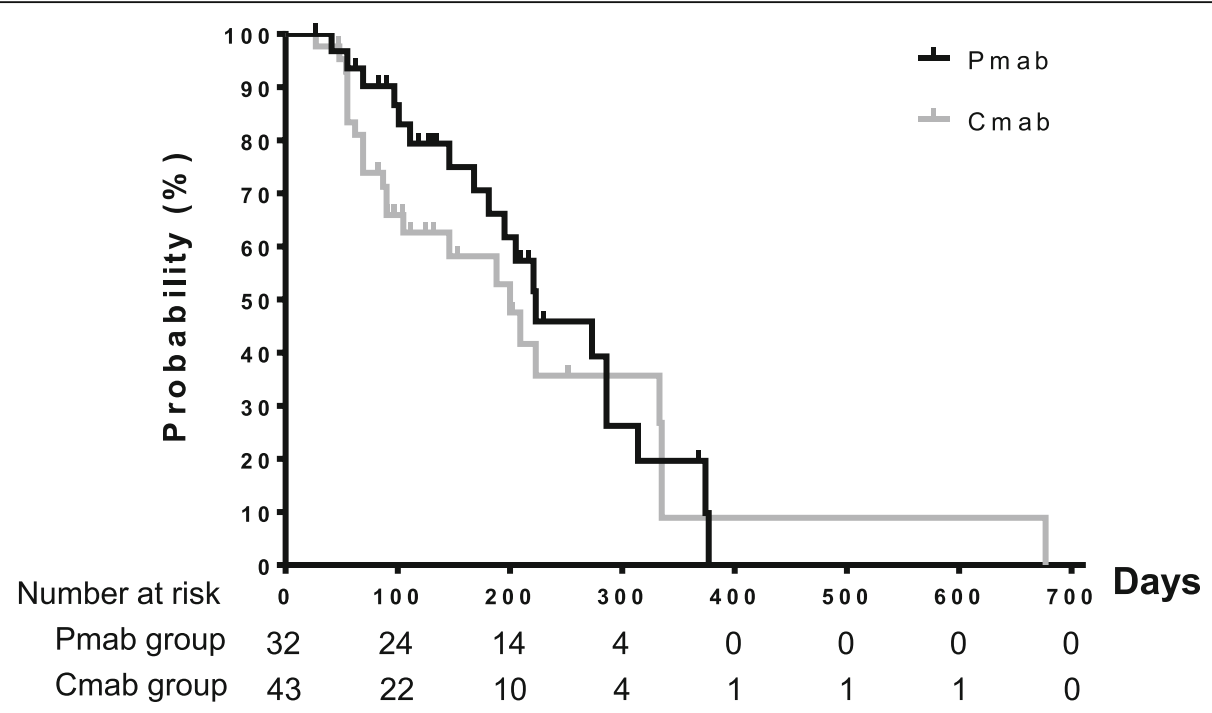

Fig. 4 Time to treatment failure between the two groups. The Kaplan-Meier curve shows the time from treatment initiation to discontinuation for any reason between the two groups. The black line represents patients in the Pmab group and the gray line represents patients in the Cmab group. Tic marks mean censored cases. The median time to treatment failure were 223 days in the Pmab group and 200 days in the Cmab group (hazard ratio $0.78,95 \% \mathrm{Cl} 0.42-1.38, P=0.39$ )

smoking status, because of the small sample size. Regarding neutropenia, which is a well-known confounder for oral mucositis, the incidence of grade 3-4 neutropenia was higher in the Cmab group. However, as the incidence of grade 2-3 oral mucositis was higher in the Pmab group, the incidence of neutropenia could not affect our study results. Second, as oral mucositis generally occurs during chemotherapy intervals, our assessment of oral toxicity mainly depended on patient interviews and patient diaries more so than the oral condition at the infusion date. Therefore, patient recall bias and interviewer bias cannot be excluded. To confirm our findings, a large prospective observational study should be conducted.

\section{Conclusions}

Pmab-based chemotherapy resulted in significantly higher grades of oral mucositis compared with Cmab-based chemotherapy. The oral condition should be monitored carefully and early supportive care should be provided for patients treated with Pmab-based chemotherapy.

\section{Additional file}

Additional file 1: Table S1. Doses and schedules of each 5-FU based chemotherapy combined with anti-EGFR antibody. (PDF $11 \mathrm{~kb}$ )

\section{Abbreviations}

5-FU: 5-fluorouracil; Cmab: Cetuximab; CTCAE: The Common Terminology Criteria for Adverse Events; EGF: Epidermal growth factor; EGFR: Epidermal growth factor receptor; FOLFIRI: Infusional 5-fluorouracil plus leucovorin with the addition of irinotecan; FOLFOX: Infusional 5-fluorouracil plus leucovorin with the addition of oxaliplatin; LV: Leucovorin; Pmab: Panitumumab; TTF: Time to treatment failure; WT: Wild type

\section{Funding}

No specific funding was received for this study.

\section{Availability of data and materials}

The datasets used and/or analysed during the current study are available from the corresponding author on reasonable request.

\section{Authors' contributions}

Conception and design of this study, analysis, and interpretation of data were performed by SD and SI. SD and KK carried out the assessment of oral mucositis. Critical revision of this article for important intellectual content was performed by DH, SN, YK and TT. All authors read and approved the final manuscript.

\section{Ethics approval and consent to participate}

This study is a retrospective cohort study, carried out by the opt-out method of a poster presentation at Kyoto-Katsura Hospital. The Ethics Committee of Kyoto-Katsura Hospital approved this retrospective study, and it was exempted from obtaining informed consent from each patient in consideration of the aim and methods of the study (Approval number: 501).

Consent for publication

Not applicable.

\section{Competing interests}

The authors declare that they have no competing interests.

\section{Publisher's Note}

Springer Nature remains neutral with regard to jurisdictional claims in published maps and institutional affiliations.

\section{Author details}

'Department of Pharmacy, Kyoto-Katsura Hospital, 17, Yamadahiraocho, Kyoto-shi Nishikyo-ku, Kyoto 615-8256, Japan. ${ }^{2}$ Department of Pharmacy, Shiga University of Medical Science Hospital, Seta Tsukinowa-cho, Otsu, Shiga 520-2192, Japan. ${ }^{3}$ Department of Pharmacy, Japanese Red Cross Kyoto Daiichi Hospital, 15-749, Hommachi, Kyoto-shi Higashiyama-ku, Kyoto 605-0981, Japan. ${ }^{4}$ College of Pharmaceutical Sciences, Ritsumeikan University, Noji-higashi 1-1-1, Kusatsu, Shiga 525-8577, Japan. 
Received: 12 August 2017 Accepted: 26 September 2018

Published online: 05 October 2018

\section{References}

1. Lalla RV, Bowen J, Barasch A, Elting L, Epstein J, Keefe DM, McGuire DB, Migliorati C, Nicolatou-Galitis O, Peterson DE, et al. MASCC/ISOO clinical practice guidelines for the management of mucositis secondary to cancer therapy. Cancer. 2014;120(10):1453-61.

2. Al-Ansari S, Zecha JA, Barasch A, de Lange J, Rozema FR, Raber-Durlacher JE. Oral mucositis induced by anticancer therapies. Curr Oral Health Rep. 2015;2(4):202-11

3. Sonis ST. A biological approach to mucositis. J Support Oncol. 2004; 2(1):21-32 discussion 35-26.

4. Cheng KK, Leung SF, Liang RH, Tai JW, Yeung RM, Thompson DR. Severe oral mucositis associated with cancer therapy: impact on oral functional status and quality of life. Support Care Cancer. 2010;18(11):1477-85.

5. Tarnawski AS, Jones MK. The role of epidermal growth factor (EGF) and its receptor in mucosal protection, adaptation to injury, and ulcer healing: involvement of EGFR signal transduction pathways. J Clin Gastroenterol. 1998;27(Suppl 1):S12-20.

6. Epstein JB, Gorsky M, Guglietta A, Le N, Sonis ST. The correlation between epidermal growth factor levels in saliva and the severity of oral mucositis during oropharyngeal radiation therapy. Cancer. 2000;89(11):2258-65.

7. Wu HG, Song SY, Kim YS, Oh YT, Lee CG, Keum KC, Ahn YC, Lee SW. Therapeutic effect of recombinant human epidermal growth factor (RhEGF) on mucositis in patients undergoing radiotherapy, with or without chemotherapy, for head and neck cancer: a double-blind placebo-controlled prospective phase 2 multi-institutional clinical trial. Cancer. 2009:115(16):3699-708.

8. Kim KI, Kim JW, Lee HJ, Kim BS, Bang SM, Kim I, Oh JM, Yoon SS, Lee JS, Park $\mathrm{S}$, et al. Recombinant human epidermal growth factor on oral mucositis induced by intensive chemotherapy with stem cell transplantation. Am J Hematol. 2013;88(2):107-12.

9. Van Cutsem E, Cervantes A, Adam R, Sobrero A, Van Krieken JH, Aderka D, Aranda Aguilar E, Bardelli A, Benson A, Bodoky G, et al. ESMO consensus guidelines for the management of patients with metastatic colorectal cancer. Ann Oncol. 2016;27(8):1386-422.

10. Zaniboni A, Formica V. The best. First. Anti-EGFR before anti-VEGF, in the first-line treatment of RAS wild-type metastatic colorectal cancer: from bench to bedside. Cancer Chemother Pharmacol. 2016;78(2):233-44

11. Patel GS, Karapetis CS. Personalized treatment for advanced colorectal cancer: KRAS and beyond. Cancer Manag Res. 2013;5:387-400.

12. Hu J, Zhang Z, Zheng R, Cheng L, Yang M, Li L, Liu B, Qian X. On-treatment markers as predictors to guide anti-EGFR MoAb treatment in metastatic colorectal cancer: a systematic review with meta-analysis. Cancer Chemother Pharmacol. 2017;79(2):275-85.

13. Fakih $M$, Vincent $M$. Adverse events associated with anti-EGFR therapies for the treatment of metastatic colorectal cancer. Curr Oncol. 2010;17(Suppl 1):S18-30.

14. Yamaguchi K, Watanabe T, Satoh T, Ishiguro M, Izawa M, Inoshiri S, Sugihara K Sakata Y. Severe infusion reactions to cetuximab occur within $1 \mathrm{~h}$ in patients with metastatic colorectal cancer: results of a nationwide, multicenter, prospective registry study of 2126 patients in Japan. Jpn J Clin Oncol. 2014;44(6):541-6.

15. Tronconi MC, Sclafani F, Rimassa L, Carnaghi C, Personeni N, Santoro A. Fata infusion reaction to cetuximab: the need for predictive risk factors and safer patient selection. J Clin Oncol. 2011;29(23):e680-1.

16. Boku N, Sugihara K, Kitagawa Y, Hatake K, Gemma A, Yamazaki N, Muro K, Hamaguchi T, Yoshino T, Yana I, et al. Panitumumab in Japanese patients with unresectable colorectal cancer: a post-marketing surveillance study of 3085 patients. Jpn J Clin Oncol. 2014;44(3):214-23.

17. Fujii $H$, lihara H, Suzuki A, Kobayashi R, Matsuhashi N, Takahashi T, Yoshida K, Itoh $Y$. Hypomagnesemia is a reliable predictor for efficacy of anti-EGFR monoclonal antibody used in combination with first-line chemotherapy for metastatic colorectal cancer. Cancer Chemother Pharmacol. 2016;77(6):1209-15.

18. Wang Q, Qi Y, Zhang D, Gong C, Yao A, Xiao Y, Yang J, Zhou F, Zhou Y. Electrolyte disorders assessment in solid tumor patients treated with antiEGFR monoclonal antibodies: a pooled analysis of 25 randomized clinical trials. Tumour Biol. 2015;36(5):3471-82.

19. Osawa M, Kudoh S, Sakai F, Endo M, Hamaguchi T, Ogino Y, Yoneoka M,

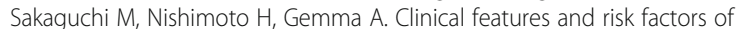
panitumumab-induced interstitial lung disease: a postmarketing all-case surveillance study. Int J Clin Oncol. 2015;20(6):1063-71.

20. Price TJ, Peeters M, Kim TW, Li J, Cascinu S, Ruff P, Suresh AS, Thomas A, Tjulandin S, Zhang $\mathrm{K}$, et al. Panitumumab versus cetuximab in patients with chemotherapy-refractory wild-type KRAS exon 2 metastatic colorectal cancer (ASPECCT): a randomised, multicentre, open-label, non-inferiority phase 3 study. Lancet Oncol. 2014;15(6):569-79.

21. Douillard JY, Siena S, Cassidy J, Tabernero J, Burkes R, Barugel M, Humblet Y, Bodoky G, Cunningham D, Jassem J, et al. Randomized, phase III trial of panitumumab with infusional fluorouracil, leucovorin, and oxaliplatin (FOLFOX4) versus FOLFOX4 alone as first-line treatment in patients with previously untreated metastatic colorectal cancer: the PRIME study. J Clin Oncol. 2010;28(31):4697-705.

22. Heinemann V, von Weikersthal LF, Decker T, Kiani A, Vehling-Kaiser U, AlBatran SE, Heintges T, Lerchenmüller C, Kahl C, Seipelt G, et al. FOLFIRI plus cetuximab versus FOLFIRI plus bevacizumab as first-line treatment for patients with metastatic colorectal cancer (FIRE-3): a randomised, openlabel, phase 3 trial. Lancet Oncol. 2014;15(10):1065-75.

23. Schwartzberg LS, Rivera F, Karthaus M, Fasola G, Canon JL, Hecht JR, Yu H, Oliner KS, Go WY. PEAK: a randomized, multicenter phase II study of panitumumab plus modified fluorouracil, leucovorin, and oxaliplatin (mFOLFOX6) or bevacizumab plus mFOLFOX6 in patients with previously untreated, unresectable, wild-type KRAS exon 2 metastatic colorectal cancer. J Clin Oncol. 2014;32(21):2240-7.

24. Peeters M, Oliner KS, Price TJ, Cervantes A, Sobrero AF, Ducreux M, Hotko Y, André T, Chan E, Lordick F, et al. Analysis of KRAS/NRAS mutations in a phase III study of Panitumumab with FOLFIRI compared with FOLFIRI alone as second-line treatment for metastatic colorectal Cancer. Clin Cancer Res. 2015;21(24):5469-79.

25. Maughan TS, Adams RA, Smith CG, Meade AM, Seymour MT, Wilson RH, Idziaszczyk S, Harris R, Fisher D, Kenny SL, et al. Addition of cetuximab to oxaliplatin-based first-line combination chemotherapy for treatment of advanced colorectal cancer: results of the randomised phase 3 MRC COIN trial. Lancet. 2011:377(9783):2103-14.

26. Peterson DE, Ohrn K, Bowen J, Fliedner M, Lees J, Loprinzi C, Mori T, Osaguona A, Weikel DS, Elad S, et al. Systematic review of oral cryotherapy for management of oral mucositis caused by cancer therapy. Support Care Cancer. 2013;21(1):327-32.

27. lihara H, Ishihara M, Matsuura K, Kurahashi S, Takahashi T, Kawaguchi Y, Yoshida K, Itoh Y. Pharmacists contribute to the improved efficiency of medical practices in the outpatient cancer chemotherapy clinic. J Eval Clin Pract. 2012;18(4):753-60.

28. Suzuki S, Chan A, Nomura H, Johnson PE, Endo K, Saito S. Chemotherapy regimen checks performed by pharmacists contribute to safe administration of chemotherapy. J Oncol Pharm Pract. 2017;23(1):18-25.

29. Dolton MJ, McLachlan AJ. Optimizing azole antifungal therapy in the prophylaxis and treatment of fungal infections. Curr Opin Infect Dis. 2014;27(6):493-500

30. Wong K, Baidoo KE, Nayak TK, Garmestani K, Brechbiel MW, Milenic DE. In vitro and in vivo pre-clinical analysis of a F(ab')(2) fragment of Panitumumab for molecular imaging and therapy of HER1 positive cancers. EJNMMI Res. 2011;(1):1.

31. Yang BB, Lum P, Chen A, Arends R, Roskos L, Smith B, Pérez Ruixo JJ. Pharmacokinetic and pharmacodynamic perspectives on the clinical drug development of panitumumab. Clin Pharmacokinet. 2010:49(11):729-40.

32. Tan AR, Moore DF, Hidalgo M, Doroshow JH, Poplin EA, Goodin S, Mauro D, Rubin EH. Pharmacokinetics of cetuximab after administration of escalating single dosing and weekly fixed dosing in patients with solid tumors. Clin Cancer Res. 2006:12(21):6517-22.

33. Yang XD, Jia XC, Corvalan JR, Wang P, Davis CG. Development of ABX-EGF, a fully human anti-EGF receptor monoclonal antibody, for cancer therapy. Crit Rev Oncol Hematol. 2001;38(1):17-23.

34. Ciardiello F, Tortora G. A novel approach in the treatment of cancer: targeting the epidermal growth factor receptor. Clin Cancer Res. 2001;7(10):2958-70.

35. Groenestege WM, Thébault $S$, van der Wijst J, van den Berg D, Janssen $R$, Tejpar S, van den Heuvel LP, van Cutsem E, Hoenderop JG, Knoers NV, et al. Impaired basolateral sorting of pro-EGF causes isolated recessive renal hypomagnesemia. J Clin Invest. 2007;117(8):2260-7.

Ready to submit your research? Choose BMC and benefit from:

- fast, convenient online submission

- thorough peer review by experienced researchers in your field

- rapid publication on acceptance

- support for research data, including large and complex data types

- gold Open Access which fosters wider collaboration and increased citations

- maximum visibility for your research: over $100 \mathrm{M}$ website views per year

At BMC, research is always in progress.

Learn more biomedcentral.com/submissions 